\title{
Statistical designs for two-color microarray experiments involving technical replication
}

\author{
Shin-Fu Tsai ${ }^{a}$, Chen-Tuo Liao ${ }^{\mathrm{a}, *}$, Feng-Shun Chai ${ }^{\mathrm{b}}$ \\ ${ }^{a}$ Division of Biometry, Institute of Agronomy, National Taiwan University, Taipei, Taiwan \\ ${ }^{\mathrm{b}}$ Institute of Statistical Science, Academia Sinica, Taipei, Taiwan
}

Received 15 September 2005; received in revised form 17 May 2006; accepted 18 May 2006

Available online 21 June 2006

\begin{abstract}
In a two-color microarray experiment, we consider the issues of determination of which mRNA samples are to be labeled with which fluorescent dye and which mRNA samples are to be hybridized together on the same slide. Specific attention is given to the test-control experiments whose primary interest lies in comparing several test treatments with a control treatment. A statistical linear model is proposed to characterize two major sources of systematic variation: the variation among distinct slides and that between fluorescent dyes. Furthermore, the possible correlation due to technical replication is also incorporated into the model. A series of A-optimal or highly efficient designs are generated from a heuristic algorithm based on the proposed model. It is shown that the obtained designs are robust not only to the variation of the correlation because of technique replication, but also to the loss of one or two slides. In addition, the comparative experiments involving technical replication are also discussed.
\end{abstract}

(c) 2006 Elsevier B.V. All rights reserved.

Keywords: A-optimality criterion; Bioinformatics; Gene expression; Differentially expressed gene

\section{Introduction}

The two-color spotted microarray is a highly effective biotechnology to measure the expression levels of many thousands of genes simultaneously, and it has become a common tool for exploring various questions in many areas of biological and medical sciences. Statistical methodology of data transformation and data analysis for such experiments has evolved rapidly in recent years. However, most of the design issues remain unexplored. On the other hand, an efficient design cannot only provide the correct answers to the scientific questions of interest, but also ensure the experimental results with the lowest variability subject to scientific and practical constraints. Hence, statistical design should also play an important role in a two-color microarray study.

Kerr and Churchill (2001) establish a connection between the statistical designs for two-color microarray experiments with classical block designs. Specifically, loop designs are recommended to replace common reference designs in the paper. Yang and Speed (2002) discuss several important design issues concerning two-color microarray experiments. Landgrebe et al. (2006) select efficient designs for factorial experiments according to the concepts of estimable contrast and E-optimality. A similar work can be found in Glonek and Solomon (2004). But in some suitable circumstances,

\footnotetext{
* Corresponding author. Tel.: +886233664760; fax: +886223620879.

E-mail address: ctliao@ntu.edu.tw (C.-T. Liao).
} 
designs that are according to their definition inadmissible may often be preferred, e.g., the inadmissible designs may be more robust to the loss of slides. However, the measured intensities among different hybridizations are assumed to be statistically independent in all the studies described above. As discussed in Speed and Yang (2002), the resulting measurements between different hybridizations could be correlated if they use a common source of mRNA extraction, known as technical replicates. In the present study, we consider a more realistic situation that the measured intensities between different hybridizations are allowed to be correlated. Specifically, we focus on the test-control experiments in which the biological interest lies in identification of differentially expressed genes between each of several test treatments versus a control treatment.

The rest of the article is organized as follows. Section 2 reviews the two-color microarray experiments. Section 3 introduces the proposed statistical linear model and the optimality criterion for choosing designs. Section 4 proposes an algorithm to search A-optimal or highly efficient designs. Section 5 discusses statistical properties of the obtained designs. Discussion and final remarks are given in the last section.

\section{Two-color microarray experiments}

The primary processes of a two-color spotted microarray can be summarized as follows. (i) Thousands of single strand cDNA sequences (probes) are robotically spotted in a high-density array on a microscope slide. (ii) Two sources of purified mRNA samples (targets) are reverse transcribed into cDNA and labeled with red fluorescent dye Cy5 and green fluorescent dye $\mathrm{Cy} 3$, respectively. (iii) These two labeled cDNA sequences are thus pooled in equal proportions and competitively hybridized with the probes on the slide. (iv) After the hybridization, fluorescent intensities of spots for each channel are separately obtained by a laser scanner at an appropriate wavelength.

There are two different types of replication in a two-color microarray experiment: (i) the biological replication is the true replication, meaning that the replicated target mRNA samples between different slides come from different biological sources. (ii) The technical replication is similar to the repeated measurement. mRNA samples are called technical replicates if they are obtained from the same mRNA extraction. The experimental results or conclusions based on the biological replication could have meaningful biological inferences. The estimation for the relative gene expression levels merely based on the technical replication usually involves smaller degree of variation. Therefore, the variability of technical replication is one of the key indices for evaluation of the quality of a two-color microarray experiment.

Most of the current design framework related to two-color microarray experiments concentrates on the comparative experiments. An experiment is called a comparative experiment if pairwise comparisons among different test treatments are of primary interest for an investigator. Namely, the investigator is interested in estimating the elementary contrasts: $\tau_{j}-\tau_{k}$ for $j \neq k$ where $\tau_{j}$ and $\tau_{k}$ denote the effects of test treatments $j$ and $k$, respectively. However, the test-control experiments are also frequently encountered in practical applications. For instance, one may be interested in detecting differentially expressed genes between distinct mutant strains and the wildtype of an organism; or between a cell line treated with different experimental conditions and without treatment (standard). Hence, the contrasts of the control versus each of the tests, i.e., $\tau_{0}-\tau_{j}$ for all $j$ are of main interest where $\tau_{0}$ denotes the effect of the control treatment.

\section{The statistical linear model and A-optimality criterion}

Suppose there are $a$ slides and $v$ test treatments with 1 control treatment in a two-color microarray experiment. For a particular spot on slide $i$, the two measured intensities can be separately characterized by the following additive models:

$$
\log _{2}\left(R_{i j}\right)=\mu_{R}+\alpha_{i}+\tau_{j}+\varepsilon_{i j}
$$

and

$$
\log _{2}\left(G_{i k}\right)=\mu_{G}+\alpha_{i}+\tau_{k}+\varepsilon_{i k}^{*}
$$

where $\log _{2}\left(R_{i j}\right)$ is the log-intensity of mRNA sample $j$ labeled with the red dye; $\log _{2}\left(G_{i k}\right)$ is the $\log$-intensity of mRNA sample $k$ labeled with the green dye; $\mu_{R}$ and $\mu_{G}$ denote the expected readings using the red and green dyes, respectively; $\alpha_{i}$ denotes the array effect of slide $i ; \tau_{j}$ and $\tau_{k}$ denote the effects of mRNA samples $j$ and $k$, respectively; $\varepsilon_{i j}$ and $\varepsilon_{i k}^{*}$ are random errors with common means zero, variances $\operatorname{Var}\left(\varepsilon_{i j}\right)=\sigma_{R}^{2}$ and $\operatorname{Var}\left(\varepsilon_{i k}^{*}\right)=\sigma_{G}^{2}$ and covariance $\operatorname{Cov}\left(\varepsilon_{i j}, \varepsilon_{i k}^{*}\right)=\sigma_{R G}$, for $i=1,2, \ldots, a$ and $0 \leqslant j \neq k \leqslant v$. 
The log-ratio of the measured readings from the two different channels, also known as M-value in the two-color microarray context, is usually recommended to represent the relative abundance of the particular gene in the two mRNA samples of interest (Yang and Speed, 2002). Hence, the difference between models (1) and (2) is given by

$$
\begin{aligned}
m_{i(j k)} & =\log _{2}\left(\frac{R_{i j}}{G_{i k}}\right) \\
& =\mu+\tau_{j}-\tau_{k}+\delta_{i(j k)},
\end{aligned}
$$

where $m_{i(j k)}$ is the log-ratio of $R_{i j}$ to $G_{i k} ; \mu=\mu_{R}-\mu_{G}$ is the dye bias effect; and $\delta_{i(j k)}$ are uncorrelated random errors with a common mean zero and $\operatorname{Var}\left(\delta_{i(j k)}\right)=\sigma_{R}^{2}+\sigma_{G}^{2}-2 \sigma_{R G}=\sigma^{2}$.

The log-ratios between different hybridizations could be correlated if they involve technical replicates. This may violate the assumption on $\delta_{i(j k)}$ in model (3). We now take into account the correlation due to a common mRNA sample involving two slides. Suppose that mRNA sample $j$ is labeled with red dye and separately cohybridized with different target samples $k$ and $h$ on slides $i$ and $i^{*}$. Then it is assumed that

$$
\operatorname{Cov}\left[\log _{2}\left(R_{i j} / G_{i k}\right), \log _{2}\left(R_{i^{*} j} / G_{i^{*} h}\right)\right]=\operatorname{Cov}\left[\log _{2}\left(R_{i j}\right), \log _{2}\left(R_{i^{*} j}\right)\right]=\eta^{2},
$$

for $j=0,1,2, \ldots, v$. Similarly, mRNA sample $j$ is labeled with green dye on the two slides,

$$
\operatorname{Cov}\left[\log _{2}\left(R_{i k} / G_{i j}\right), \log _{2}\left(R_{i^{*} h} / G_{i^{*} j}\right)\right]=\operatorname{Cov}\left[-\log _{2}\left(G_{i j}\right),-\log _{2}\left(G_{i^{*} j}\right)\right]=\eta^{2} .
$$

Also, mRNA sample $j$ is labeled with distinct dyes on the two slides,

$$
\operatorname{Cov}\left[\log _{2}\left(R_{i j} / G_{i k}\right), \log _{2}\left(R_{i^{* h}} / G_{i^{*} j}\right)\right]=\operatorname{Cov}\left[\log _{2}\left(R_{i j}\right),-\log _{2}\left(G_{i^{*} j}\right)\right]=-\eta^{2},
$$

and

$$
\operatorname{Cov}\left[\log _{2}\left(R_{i k} / G_{i j}\right), \log _{2}\left(R_{i^{* j}} / G_{i * h}\right)\right]=\operatorname{Cov}\left[-\log _{2}\left(G_{i j}\right), \log _{2}\left(R_{i^{*} j}\right)\right]=-\eta^{2} .
$$

The above assumptions lead to that the covariances between a pair of dye-swap and replicated hybridizations are equal to $-2 \eta^{2}$ and $2 \eta^{2}$, respectively. Namely,

$$
\begin{aligned}
\operatorname{Cov}\left[\log _{2}\left(R_{i j} / G_{i k}\right), \log _{2}\left(R_{i^{*} k} / G_{i^{*}}\right)\right]= & \operatorname{Cov}\left[\log _{2}\left(R_{i j}\right),-\log _{2}\left(G_{i^{*} j}\right)\right] \\
& +\operatorname{Cov}\left[-\log _{2}\left(G_{i k}\right), \log _{2}\left(R_{i^{*} k}\right)\right] \\
= & -2 \eta^{2}
\end{aligned}
$$

and

$$
\begin{aligned}
\operatorname{Cov}\left[\log _{2}\left(R_{i j} / G_{i k}\right), \log _{2}\left(R_{i^{*} j} / G_{i * k}\right)\right]= & \operatorname{Cov}\left[\log _{2}\left(R_{i j}\right), \log _{2}\left(G_{i^{*} j}\right)\right] \\
& +\operatorname{Cov}\left[-\log _{2}\left(G_{i k}\right),-\log _{2}\left(R_{i^{*} k}\right)\right] \\
= & 2 \eta^{2},
\end{aligned}
$$

for $0 \leqslant j \neq k \leqslant v$. In consequence, we may modify the assumption on $\delta_{i(j k)}$ of model (3) as follows. For slides $i$ and $i^{*}$, the covariance between $\delta_{i(j k)}$ and $\delta_{i^{*}(g h)}$ is defined by

$$
\operatorname{Cov}\left[\delta_{i(j k)}, \delta_{i^{*}(g h)}\right]= \begin{cases}\sigma^{2} & \text { if } i=i^{*}, \\ \rho \sigma^{2} & \text { if } i \neq i^{*} \text { and } j=g, k \neq h \text { or } j \neq g, k=h, \\ 2 \rho \sigma^{2} & \text { if } i \neq i^{*}, j=g, k=h, \\ -\rho \sigma^{2} & \text { if } i \neq i^{*} \text { and } j=h, k \neq g \text { or } j \neq h, k=g, \\ -2 \rho \sigma^{2} & \text { if } i \neq i^{*}, j=h, k=g, \\ 0 & \text { otherwise, }\end{cases}
$$


where $\rho=\eta^{2} / \sigma^{2}$, the correlation due to two technical replicates labeled with one identical fluorescent dye. Hence, the measured log-ratios (M-values) based on the modified model can be expressed in the following matrix form:

$$
\boldsymbol{m}=\mathbf{1}_{a} \mu+\boldsymbol{X} \tau+\boldsymbol{\delta},
$$

where $\boldsymbol{m}$ is the $a \times 1$ vector whose entries are log-ratios; $\mathbf{1}_{a}$ is the $a \times 1$ vector with all entries equal to $1 ; \tau=$ $\left[\tau_{0}, \tau_{1}, \tau_{2}, \ldots, \tau_{v}\right]^{\prime}$ is the vector of treatment effects; and $\boldsymbol{X}$ is an $a \times(v+1)$ matrix whose entries, depending on the choice of experimental design, can be defined by

$$
x_{i j}= \begin{cases}1 & \text { if mRNA sample } j \text { labeled with red fluorescent dye on slide } i, \\ -1 & \text { if mRNA sample } j \text { labeled with green fluorescent dye on slide } i, \\ 0 & \text { otherwise. }\end{cases}
$$

Also the covariance matrix of $\delta$ is determined by (4).

A two-color microarray design can be described by a set of ordered pairs. Two treatments appearing in the same parentheses mean that these two treatments are hybridized together on the same slide. The treatment occurs in the first (second) position is labeled with red (green) fluorescent dye. For instance, $(0,1)$ is the slide in which the control treatment and test treatment 1 are labeled with red and green fluorescent dyes, respectively.

Now suppose that an investigator hopes to screen out the differentially expressed genes between each of three test treatments and one control treatment, and eight slides are available for the experiment. Design

$$
d=\{(0,1),(0,2),(0,3),(1,0),(2,0),(3,0),(1,3),(3,2)\}
$$

is chosen to conduct the experiment. Then the measured log-ratios can be represented by the proposed model as follows:

$$
\left[\begin{array}{l}
m_{1(0,1)} \\
m_{2(0,2)} \\
m_{3(0,3)} \\
m_{4(1,0)} \\
m_{5(2,0)} \\
m_{6(3,0)} \\
m_{7(1,3)} \\
m_{8(3,2)}
\end{array}\right]=\left[\begin{array}{l}
1 \\
1 \\
1 \\
1 \\
1 \\
1 \\
1 \\
1
\end{array}\right] \mu+\left[\begin{array}{cccc}
1 & -1 & 0 & 0 \\
1 & 0 & -1 & 0 \\
1 & 0 & 0 & -1 \\
-1 & 1 & 0 & 0 \\
-1 & 0 & 1 & 0 \\
-1 & 0 & 0 & 1 \\
0 & 1 & 0 & -1 \\
0 & 0 & -1 & 1
\end{array}\right]\left[\begin{array}{c}
\tau_{0} \\
\tau_{1} \\
\tau_{2} \\
\tau_{3}
\end{array}\right]+\left[\begin{array}{l}
\delta_{1(0,1)} \\
\delta_{2(0,2)} \\
\delta_{3(0,3)} \\
\delta_{4(1,0)} \\
\delta_{5(2,0)} \\
\delta_{6(3,0)} \\
\delta_{7(1,3)} \\
\delta_{8(3,2)}
\end{array}\right] .
$$

The covariance matrix of the log-ratios is given by

$$
\boldsymbol{V}=\sigma^{2}\left[\begin{array}{cccccccc}
1 & \rho & \rho & -2 \rho & -\rho & -\rho & -\rho & 0 \\
\rho & 1 & \rho & -\rho & -2 \rho & -\rho & 0 & \rho \\
\rho & \rho & 1 & -\rho & -\rho & -2 \rho & \rho & -\rho \\
-2 \rho & -\rho & -\rho & 1 & \rho & \rho & \rho & 0 \\
-\rho & -2 \rho & -\rho & \rho & 1 & \rho & 0 & -\rho \\
-\rho & -\rho & -2 \rho & \rho & \rho & 1 & -\rho & \rho \\
-\rho & 0 & \rho & \rho & 0 & -\rho & 1 & -\rho \\
0 & \rho & -\rho & 0 & -\rho & \rho & -\rho & 1
\end{array}\right] .
$$

The normal equations for the best linear unbiased estimators (BLUEs) of $\mu$ and $\tau$, denoted by $\hat{\mu}$ and $\hat{\tau}$, according to the generalized least-square method are given by

$$
\mathbf{1}_{a}^{\prime} \boldsymbol{V}^{-1} \mathbf{1}_{a} \hat{\mu}+\mathbf{1}_{a}^{\prime} \boldsymbol{V}^{-1} \boldsymbol{X} \hat{\tau}=\mathbf{1}_{a}^{\prime} \boldsymbol{V}^{-1} \boldsymbol{m}
$$


and

$$
\boldsymbol{X}^{\prime} \boldsymbol{V}^{-1} \mathbf{1}_{a} \hat{\mu}+\boldsymbol{X}^{\prime} \boldsymbol{V}^{-1} \boldsymbol{X} \hat{\tau}=\boldsymbol{X}^{\prime} \boldsymbol{V}^{-1} \boldsymbol{m} .
$$

It follows that the reduced information matrix of $\tau$ is given by

$$
\boldsymbol{M}=\boldsymbol{X}^{\prime} \boldsymbol{V}^{-1} \boldsymbol{X}-\boldsymbol{X}^{\prime} \boldsymbol{V}^{-1} \mathbf{1}_{a}\left(\boldsymbol{I}_{a}^{\prime} \boldsymbol{V}^{-1} \mathbf{1}_{a}\right)^{-1} \mathbf{1}_{a}^{\prime} \boldsymbol{V}^{-1} \boldsymbol{X}
$$

Furthermore, let $\overline{\boldsymbol{M}}$ denote the information matrix for the control versus test contrasts: $\tau_{0}-\tau_{j}$ for $j=1,2, \ldots, v$. Then $\bar{M}$ can just be obtained by eliminating the first row and the first column of $\boldsymbol{M}$ of (6) (Bechhofer and Tamhane, 1981). A test-control design is said to be connected if and only if the rank of its information matrix $\bar{M}$ is equal to $v$. A connected design guarantees that all the control versus test contrasts are estimable. Let $0<\lambda_{1} \leqslant \lambda_{2} \leqslant \cdots \leqslant \lambda_{v}$ denote the eigenvalues of information matrix $\bar{M}$ for a connected design. Then the A-optimality score (A-score for simplicity) of the design is defined by

$$
\text { A-score }=\sum_{i=1}^{v} \frac{1}{\lambda_{i}} .
$$

It is easy to verify that the A-score is exactly equal to the average variance of the BLUEs of $\tau_{0}-\tau_{j}$ for $j=1,2, \ldots, v$. A design minimizing the A-score is called A-optimal. In this paper, we provide an algorithm for searching the A-optimal design among a class of competing designs.

\section{Algorithm}

In this study, we only consider the designs in which the same mRNA samples are not allowed to be cohybridized on the same slide. It can be shown that the reduced information matrix of treatment effects based on model (3) is exactly equal to twice of that based on the ANOVA model of the classical row-column design. A proof of this result is given in Appendix A. Therefore, designing a two-color microarray experiment with $a$ slides and $v+1$ mRNA samples is equivalent to choosing a classical $2 \times a(v+1)$-treatment row-column design, where the two rows correspond to the two dyes, the columns are the slides and the treatments are the mRNA samples. Consequently, we can focus on the A-optimal design within the class of $2 \times a(v+1)$-treatment row-column designs, where $v+1$ consists of $v$ test treatments and 1 control treatment.

The true value of $\rho$ due to technical replication is usually unknown at the planning stage of an experiment. However, Lemma 1 shows that the possible range of $\rho$ is from 0 to 0.5. A proof of the lemma can be found in Appendix B.

Lemma 1. Suppose that the covariance matrix of measured log-ratios of an experiment is positive definite. For any connected $2 \times a(v+1)$-treatment row-column design, where $a \geqslant v+1$, then $0 \leqslant \rho<0.5$ under the covariance structure described as (4).

Notice that it is easy to see that $0 \leqslant \rho<0.5$ if there is a pair of dye-swap or replicated hybridizations in a design. However, most of the connected designs may contain neither of these two pairs. For fixed $v, a$ and a specified value of $\rho$, the following algorithm provides a method of systematically searching A-optimal or nearly A-optimal designs for the test-control experiments. The algorithm is composed of three operations described below.

Operation (1): Using the free software "geng" (McKay, 1991) to generate all non-isomorphic connected simple graphs with $n$ nodes and $e$ edges. In a simple graph, each pair of nodes of an edge can be regarded as two mRNA samples cohybridized on a slide. This operation provides all possible connected block designs of size two for the fixed number of treatments $n$ and number of blocks $e$. Let $G(n, e)$ denote the set of all possible designs generated from this operation for given $n$ and $e$.

Operation (2): For each design in $G(n, e)$, decide which of the two treatments in each column appears in the first row (labeled with red dye) and which appears in the second row (labeled with green dye). Let $r_{R_{j}}$ and $r_{G_{j}}$ denote the numbers of treatment $j$ appearing in the first and second rows, respectively, and $r_{j}=r_{R_{j}}+r_{G_{j}}$, for $j=1,2, \ldots, n$. Rearrange the treatments in each column such that $r_{R_{j}}=r_{G_{j}}$ if $r_{j}$ is even and $r_{R_{j}}=r_{G_{j}}+1$ or $r_{G_{j}}=r_{R_{j}}+1$ if $r_{j}$ is 
odd. Let $\mathrm{RCD}(n, e)$ denote the collection of all possible row-column designs generated from this operation for a given $G(n, e)$.

Operation (3): For a design of $\operatorname{RCD}(n, e)$, each treatment can be considered as the control treatment, resulting in $n$ possible test-control designs. For a specified value of $\rho$, compute the information matrix $\boldsymbol{M}$ of (6) and delete the $i$ th row and the $i$ th column of $\boldsymbol{M}$ to obtain the information matrix for the control versus test contrasts when treatment $i$ is regarded as the control. Denote this information matrix as $\overline{\boldsymbol{M}}_{i}$. Repeat this for $i=1,2, \ldots, n$ to give $\overline{\boldsymbol{M}}_{1}, \overline{\boldsymbol{M}}_{2}, \ldots, \overline{\boldsymbol{M}}_{n}$. Check the connectedness of these designs and calculate their A-scores defined in (7). Select the design with minimal A-score among all possible connected designs generated from $\operatorname{RCD}(n, e)$ and denote it by $d^{*}$.

For a pre-specified number of slides, say $a$, the algorithm for searching A-optimal or nearly A-optimal designs for the test-control experiments is described as follows.

(A) When $v+1 \leqslant a \leqslant 2 v-1$, the algorithm carries out the following steps:

Step 1: Perform Operation (1) to get $G(v+1, a)$.

Step 2: Perform Operation (2) to get $\operatorname{RCD}(v+1, a)$.

Step 3: For $\rho=0,0.1,0.2,0.3,0.4,0.49$, perform Operation (3) to get the required designs $d^{*}$ s.

(B) When $2 v \leqslant a \leqslant\left(\begin{array}{c}v+1 \\ 2\end{array}\right)$, the algorithm carries out the following steps:

Step 1: Conduct Steps 1-3 in (A) to obtain designs $d^{*}$ s.

Step 2: Define the direct-comparison dye-swap design (dcdsd) by

$$
\operatorname{dcdsd}(v+1,2 v)=\{(0,1),(1,0),(0,2),(2,0), \ldots,(0, v),(v, 0)\},
$$

which consists of the $2 v$ slides that are the $v$ dye-swap pairs between the control and each of the test treatments. Perform Operations (1) and (2) to obtain $\operatorname{RCD}(v, a-2 v)$. Note that nodes $1,2, \ldots, v$ of each design in $\operatorname{RCD}(v, a-2 v)$ precisely correspond to the $v$ test treatments. Let the $\operatorname{dcdsd}(v+1,2 v)$ of (8) be augmented with each design of $\operatorname{RCD}(v, a-2 v)$ and denote the collection of all the augmented designs by $\operatorname{RCD}-\operatorname{dcdsd}(v+1, a)$. For each value of $\rho=0,0.1,0.2,0.3$, $0.4,0.49$, search the design with the minimal A-score among the $\operatorname{RCD}-\operatorname{dcdsd}(v+1, a)$ and denote it as $\xi^{*}$ s.

Step 3: Compare $d^{*}$ and $\xi^{*}$ for each specified value of $\rho$ and select the design with smaller A-score.

(C) When $\left(\begin{array}{c}v+1 \\ 2\end{array}\right)+1 \leqslant a \leqslant 2 v+\left(\begin{array}{c}v \\ 2\end{array}\right)$, perform the same procedure of Step 2 described in (B) to obtain the required designs $\xi^{*}$ s.

\section{Properties of the obtained designs}

We generate designs from the algorithm for $2 \leqslant v \leqslant 7$ and $v+1 \leqslant a \leqslant 2 v+\left(\begin{array}{l}v \\ 2\end{array}\right)$. In the following, we discuss some interesting properties of the obtained designs.

For a pair of fixed values of $v$ and $a$, all the designs obtained for $\rho=0,0.1,0.2,0.3,0.4,0.49$ are identical. This implies that the optimal design based on a fixed value of $\rho$ may also be optimal for a large number of values ranging from 0 to 0.5 . In reality, the true value of $\rho$ is usually unknown before conducting an experiment, so the designs robust against the variation of $\rho$ are highly desired in practical use. The obtained designs are tabulated in Appendix C.

A loss of slides can result in a loss of the estimable comparisons of interest. That is the "flawed" design can turn out to be disconnected in which the rank of information matrix $\overline{\boldsymbol{M}}$ is less than $v$. For each of the obtained designs in Appendix $\mathrm{C}$, we may investigate the two situations that any one or two slides are missing. The numbers of disconnected designs are separately reported among all possible the $\left(\begin{array}{l}a \\ 1\end{array}\right)$ and $\left(\begin{array}{l}a \\ 2\end{array}\right)$ "flawed" designs for these two situations. It can be found that almost all the obtained designs in Appendix $\mathrm{C}$, except the few with $a$ slightly larger than $v$, can still ensure that all the control versus test comparisons are estimable even one or two slides are missing. In practice, we would encourage investigators to use at least $2 v$ slides for $v$ test treatments to avoid a loss of the estimable control versus test comparisons once two slides are missing.

In general, for fixed $v$ and $\rho$, the A-score of the resulting designs decreases as the number of slides $a$ increases. In other words, the design efficiency increases as $a$ increases, but the improvement gets less significant as $a$ approaches to $2 v+\left(\begin{array}{l}v \\ 2\end{array}\right)$. In addition, when the correlation coefficient $\rho$ is fixed at 0.49 (close to the upper bound 0.5), the A-score converges to $v$ regardless of the number of slides $a$. This implies that the improvement of efficiency of the resulting designs is negligible as $a$ increases under this specific situation. Fig. 1 displays the A-score of the resulting designs for $v=6$. In the legend of the figure, the different shapes denote the specified values of $\rho$. 


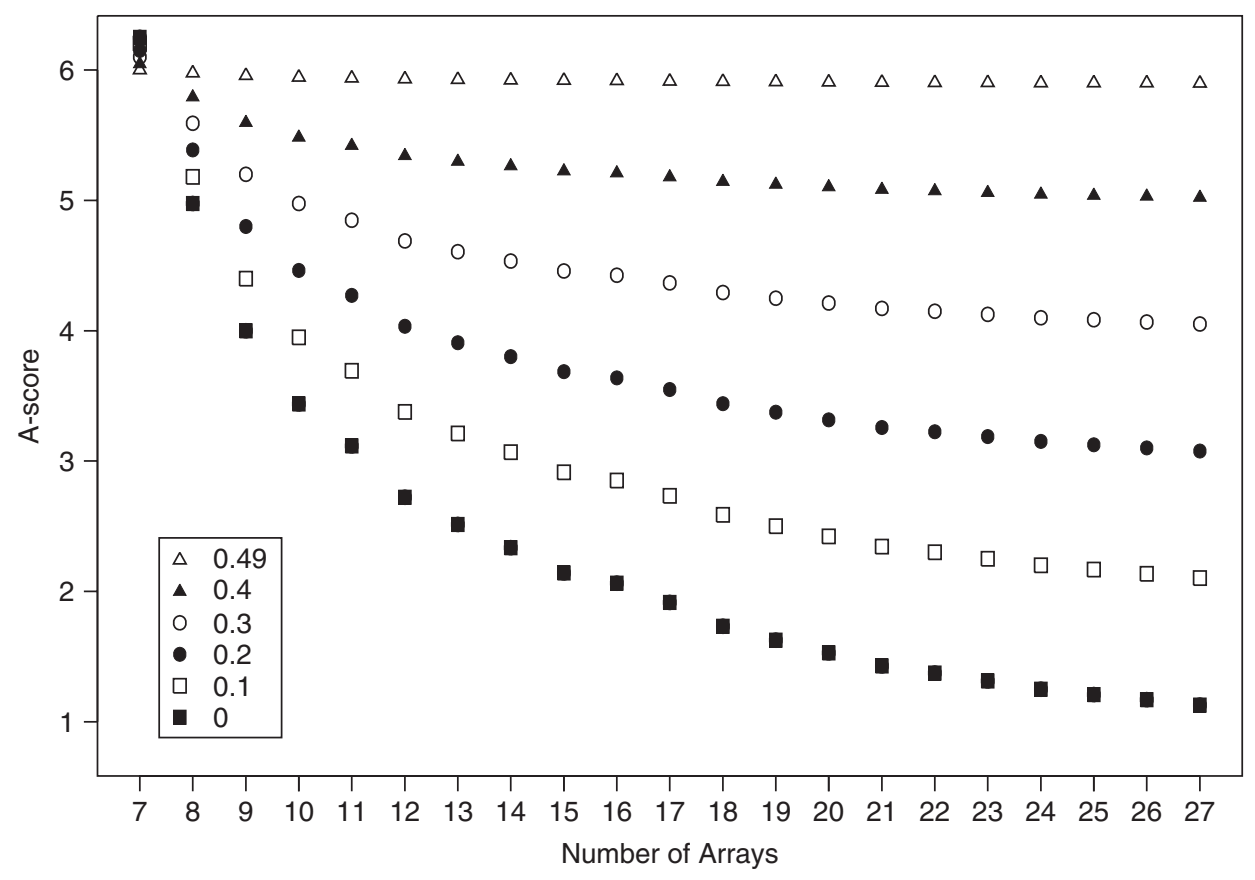

Fig. 1. A-score of the obtained designs for $v=6$.

Scientists may need information on which comparisons can be estimated most accurately. So we also report the ratios of maximum to minimum variance among the comparisons of interest for $\rho=0$ and 0.49 for each of the obtained designs in Appendix C. Due to the nature of A-optimality that minimizes the average variance of all comparisons of interest, almost all the ratios are close to 1 .

Most of the obtained designs contain either a direct-comparison dye-swap design defined as (8) or a direct-comparison design which consists of the $v$ hybridizations between each of the test treatments and the control treatment. This supports that the obtained designs could be highly efficient in estimating the control versus test comparisons of interest, because these comparisons can be made via a chain of connections involving no more than two. Specifically, we classify some of the obtained designs with equal replicates on test treatments $\left(r_{1}=r_{2}=\cdots=r_{v}\right)$ into the following design categories.

Category I: All the designs with $a=3 v$ consist of a direct-comparison dye-swap design and a loop design for $v$ test treatments.

Category II: The designs with parameters $(v=4, a=12)$ and $(v=6, a=15)$ can be identified as group divisible treatments designs (GDTDs) regarding the columns (slides) as blocks, and the rows (dyes) are all balanced, i.e., $r_{R_{0}}=r_{G_{0}}$ and $r_{R_{j}}=r_{G_{j}}$ for $j=1,2, \ldots, v$. The definition of GDTDs can be found in Jacroux (1989).

Category III: The designs with parameters $(v=4, a=8)$ and $(v=6, a=21)$ can be identified as GDTDs regarding the columns as blocks, $r_{R_{0}}=r_{G_{0}}$ and the difference of $r_{R_{j}}$ and $r_{G_{j}}$ is exactly equal to one, i.e., $\left|r_{R_{j}}-r_{G_{j}}\right|=1$ for $j=1,2, \ldots, v$.

Category IV: The designs with an odd $v$ and $a=2 v+\left(\begin{array}{l}v \\ 2\end{array}\right)$ are balanced treatments row-column designs (BTRCDs) whose definition can be found in Ture (1994).

Category V: The designs with an even $v$ and $a=2 v+\left(\begin{array}{l}v \\ 2\end{array}\right)$ are balanced treatments incomplete block designs (BTIBDs) regarding the columns as blocks. Also $r_{R_{0}}=r_{G_{0}}$ and $\left|r_{R_{j}}-r_{G_{j}}\right|=1$ for $j=1,2, \ldots, v$.

Notice that the designs with parameters $(v=4, a=12)$ and $(v=6, a=15)$ can be verified as the A-optimal designs over all possible competing designs when $\rho=0$ (Jacroux, 1989).

\section{Concluding remarks}

The test-control experiments could be sometimes confused with the common reference designs in the comparative experiments. In a common reference design, although each treatment is competitively hybridized with a common 
reference sample, the comparisons between treatments and the common reference sample are usually of no interest. The optimal design issues concerning test-control experiments have been extensively studied, the reader may refer to a review article by Hedayat et al. (1988). But there appears to be no work that specifically accommodates to the two-color microarray context.

There is a technical problem that needs to be overcome for the proposed algorithm in Section 4. The number of candidate designs become quite large when $v$ is greater than nine and this makes our computer program computationally infeasible. A MATLAB computer program for implementing the algorithm is available from the authors upon request.

One may argue that the true value of $\rho$ could be different from one spot to another. Namely, the correlation due to technical replication could be different among distinct genes. So this phenomenon should be considered in the procedure of constructing designs. Recall that the proposed statistical linear model is actually a gene-by-gene model and it has been shown that the obtained designs are robust against the variation of $\rho$. Hence, the obtained designs are anticipated to remain highly efficient even the value of $\rho$ varies among genes.

The procedures presented in this article can be adapted for the comparative experiments. Similarly, we consider the situations that the values of $\rho$ are fixed at $0,0.1,0.2,0.3,0.4$ and 0.49 and construct A-optimal or highly efficient designs using a similar algorithm of Section 4 . The resulting designs share the same robustness property as the test-control experiments. Both of the obtained designs for test-control and comparative experiments with the graphical representation recommended by Kerr and Churchill (2001) are available at website http://homepage.agron.ntu.edu.tw/ LiaoLab/.

\section{Acknowledgments}

The authors thank an associate editor and a referee for their constructive suggestions and comments that resulted in a much improved article. Thanks are also due to Professor Jen-Pei Liu of National Taiwan University for his assistance in editing.

\section{Appendix A. A connection between two-color microarray designs and classical row-column designs}

The matrix representation of model (3) is exactly the same as model (5), except the covariance matrix of $\boldsymbol{\delta}$ is changed to be $\sigma^{2} \boldsymbol{I}$, where $\boldsymbol{I}$ denotes the identity matrix. Hence, the reduced information matrix of $\tau$ can be obtained from replacing $\boldsymbol{V}$ by $\boldsymbol{I}$ in (6) and is given by

$$
\boldsymbol{F}=\boldsymbol{X}^{\prime} \boldsymbol{X}-\frac{1}{a} \boldsymbol{X}^{\prime} \mathbf{1}_{a} \mathbf{1}_{a}^{\prime} \boldsymbol{X}
$$

It is easy to verify that $\boldsymbol{X}^{\prime} \boldsymbol{X}=\boldsymbol{r}^{\delta}-\boldsymbol{\Lambda}$, where $\boldsymbol{r}^{\delta}$ is the diagonal matrix with the $j$ th diagonal element equal to $r_{j}$, the number of occurrences of mRNA sample $j$ in the design, and the $(v+1) \times(v+1)$ matrix $\boldsymbol{\Lambda}$ consists of elements $\lambda_{j k}$, the number of slides on which mRNA samples $j$ and $k$ are hybridized together. Also, let $\boldsymbol{X}^{\prime} \mathbf{1}_{a}=\boldsymbol{q}=\left[q_{0}, q_{1}, \ldots, q_{v}\right]^{\prime}$; then $q_{j}=r_{R j}-r_{G j}$, where $r_{R j}$ and $r_{G j}$ are the numbers of occurrences of mRNA sample $j$ labeled with red dye and green dye, respectively. Therefore, $\boldsymbol{F}$ can be rewritten as

$$
\boldsymbol{F}=\boldsymbol{r}^{\delta}-\boldsymbol{\Lambda}-\frac{1}{a} \boldsymbol{q} \boldsymbol{q}^{\prime}
$$

Moreover, it is easy to check that $\boldsymbol{\Lambda}=\boldsymbol{G} \boldsymbol{G}^{\prime}-\boldsymbol{r}^{\delta}$ and that $\boldsymbol{q} \boldsymbol{q}^{\prime}=2 \boldsymbol{H} \boldsymbol{H}^{\prime}-\boldsymbol{r} \boldsymbol{r}^{\prime}$, where $\boldsymbol{G}$ is the $(v+1) \times a$ treatment-slide incidence matrix, $\boldsymbol{H}$ is the $(v+1) \times 2$ treatment-dye incidence matrix, and $\boldsymbol{r}=\left[r_{0}, r_{1}, \ldots, r_{v}\right]^{\prime}$. Hence, we can also rewrite $\boldsymbol{F}$ as

$$
\boldsymbol{F}=2\left(\boldsymbol{r}^{\delta}-\frac{1}{2} \boldsymbol{G} \boldsymbol{G}^{\prime}-\frac{1}{a} \boldsymbol{H} \boldsymbol{H}^{\prime}+\frac{1}{2 a} \boldsymbol{r} \boldsymbol{r}^{\prime}\right)
$$

where the part $\left(\boldsymbol{r}^{\delta}-\frac{1}{2} \boldsymbol{G} \boldsymbol{G}^{\prime}-(1 / a) \boldsymbol{H} \boldsymbol{H}^{\prime}+(1 / 2 a) \boldsymbol{r} \boldsymbol{r}^{\prime}\right)$ in the right-hand side of the above equation is exactly the reduced information matrix of $\tau$ for the classical row-column design with two rows, $a$ columns and $v+1$ treatments. 


\section{Appendix B. Proof of Lemma 1}

Suppose that $d$ is a connected $2 \times a(v+1)$-treatment row-column design, where $a \geqslant v+1$. The proof is mainly based on the fact that $d$ must contain a subset $d_{S}$ with $k$ treatments and $k$ slides, where $k \leqslant v+1$. Let treatments $i_{1}<i_{2}<\cdots<i_{k}$ be the treatments in $d_{S}$. Then $d_{S}$ consists of the slides: $\left[i_{1}, i_{2}\right],\left[i_{2}, i_{3}\right], \ldots,\left[i_{k-1}, i_{k}\right],\left[i_{k}, i_{1}\right]$. Here, the square brackets denote unordered pairs, meaning that the dye labeling is not determined as ordered pairs. Recall that we use parentheses to denote ordered pairs in this study.

Let the loop design $d_{L}=\left\{\left(i_{1}, i_{2}\right),\left(i_{2}, i_{3}\right), \ldots,\left(i_{k-1}, i_{k}\right),\left(i_{k}, i_{1}\right)\right\}$. Also let $\boldsymbol{m}_{L}$ and $\boldsymbol{V}\left(\boldsymbol{m}_{L}\right)$ be the log-ratio vector and the covariance matrix of $d_{L}$, respectively. Similarly, let $\boldsymbol{m}_{S}$ and $\boldsymbol{V}\left(\boldsymbol{m}_{S}\right)$ for $d_{S}$. Then there is a $k \times k$ diagonal matrix $\boldsymbol{D}$ such that $\boldsymbol{m}_{S}=\boldsymbol{D} \boldsymbol{m}_{L}$, where the $j$ th diagonal element of $\boldsymbol{D}$ is 1 if slide $j$ in $d_{S}$ is $\left(i_{j}, i_{j+1}\right)$; otherwise is -1 if slide $j$ in $d_{S}$ is $\left(i_{j+1}, i_{j}\right)$, for $j=1,2, \ldots, k$. Note that $i_{k+1}=i_{1}$.

From the covariance structure of (4), $\rho \geqslant 0$ and $\boldsymbol{V}\left(\boldsymbol{m}_{L}\right)$ is a circulant matrix that can be simply represented by its first row as $\boldsymbol{V}\left(\boldsymbol{m}_{L}\right)=\operatorname{circ}[1,-\rho, 0, \ldots, 0,-\rho]$. Moreover, from Graybill (1983), the eigenvalues of $\boldsymbol{V}\left(\boldsymbol{m}_{L}\right)$ are given by

$$
\mu_{i}=1+(-\rho) w_{i}+(0) w_{i}^{2}+\cdots+(0) w_{i}^{k-2}+(-\rho) w_{i}^{k-1},
$$

for $i=1,2, \ldots, k$. Here, $w_{1}, w_{2}, \ldots, w_{k}$ are the $k$ roots of unity. Specifically, $\mu_{1}=1-2 \rho$ for $w_{1}=1$.

The proof now follows from the assumption that the covariance matrix of $d$ is positive definite, leading to that $\boldsymbol{V}\left(\boldsymbol{m}_{S}\right)$ is positive definite since it is the matrix corresponding to a principal minor of the covariance matrix of $d$. In consequence, $\boldsymbol{V}\left(\boldsymbol{m}_{L}\right)$ is also positive definite due to that $\boldsymbol{m}_{S}=\boldsymbol{D} \boldsymbol{m}_{L}$. This ensures that $\mu_{1}=1-2 \rho>0$. So $0 \leqslant \rho<0.5$.

\section{Appendix C. The robust A-optimal designs for $2 \leqslant v \leqslant 7$ and $v+1 \leqslant a \leqslant 2 v+\left(\begin{array}{c}v \\ 2\end{array}\right)$}

Note that nodd 1 and nodd 2 denote the numbers of disconnected designs among all possible the $\left(\begin{array}{l}a \\ 1\end{array}\right)$ and $\left(\begin{array}{l}a \\ 2\end{array}\right)$ "flawed" designs for the situations that one and two slides are missing, respectively. Also, rmtm0 and rmtm1 separately denote the ratios of maximum to minimum variance among the control versus test comparisons for $\rho=0$ and $\rho=0.49$.

\begin{tabular}{llllll}
\hline$a$ & Design & nodd1 & nodd2 & rmtm0 & rmtm1 \\
\hline$v=2$ & & & & & \\
3 & $(0,1),(2,0),(1,2)$ & 0 & $3^{\mathrm{a}}$ & 1.000 & 1.000 \\
4 & $(0,1),(0,2),(1,0),(2,0)$ & 0 & 2 & 1.000 & 1.000 \\
5 & $(0,1),(0,2),(1,0),(2,0),(1,2)$ & 0 & 0 & 1.000 & 1.000 \\
\hline
\end{tabular}

\begin{tabular}{llllll}
\hline$a$ & Design & nodd1 & nodd2 & rmtm0 & rmtm1 \\
\hline$v=3$ & & & & & \\
4 & $(0,1),(2,0),(1,3),(3,2)$ & 0 & $6^{\mathrm{a}}$ & 1.333 & 1.005 \\
5 & $(0,1),(2,0),(3,0),(1,3),(3,2)$ & 0 & 2 & 1.150 & 1.002 \\
6 & $(0,1),(0,2),(0,3),(1,0),(2,0),(3,0)$ & 0 & 3 & 1.000 & 1.000 \\
7 & $(0,1),(0,2),(0,3),(1,0),(2,0),(3,0),(1,3)$ & 0 & 1 & 1.300 & 1.002 \\
8 & $(0,1),(0,2),(0,3),(1,0),(2,0),(3,0),(1,3),(3,2)$ & 0 & 0 & 1.273 & 1.002 \\
9 & $(0,1),(0,2),(0,3),(1,0),(2,0),(3,0),(1,2),(2,3),(3,1)$ & 0 & 0 & 1.000 & 1.000 \\
\hline
\end{tabular}




\begin{tabular}{clllll}
\hline$a$ & Design & nodd1 & nodd2 & rmtm0 & rmtm1 \\
\hline$v=4$ & & & & & \\
5 & $(0,1),(2,0),(3,0),(1,4),(4,2)$ & 1 & $10^{\mathrm{a}}$ & 1.667 & 1.010 \\
6 & $(0,1),(0,2),(3,0),(4,0),(1,3),(2,4)$ & 0 & 6 & 1.000 & 1.000 \\
7 & $(0,1),(0,2),(3,0),(4,0),(1,4),(2,4),(4,3)$ & 0 & 3 & 1.500 & 1.004 \\
8 & $(0,1),(0,2),(3,0),(4,0),(1,3),(1,4),(2,3),(2,4)$ & 0 & 0 & 1.000 & 1.000 \\
9 & $(0,1),(0,2),(3,0),(4,0),(1,3),(2,3),(2,4),(3,4),(4,1)$ & 0 & 0 & 1.200 & 1.002 \\
10 & $(0,1),(0,2),(0,3),(0,4),(1,0),(2,0),(3,0),(4,0),(1,3),(2,4)$ & 0 & 0 & 1.000 & 1.000 \\
11 & $(0,1),(0,2),(0,3),(0,4),(1,0),(2,0),(3,0),(4,0),(1,3),(2,4)$, & 0 & 0 & 1.282 & 1.002 \\
& $(4,1)$ & & & & \\
12 & $(0,1),(0,2),(0,3),(0,4),(1,0),(2,0),(3,0),(4,0),(1,2),(2,3)$, & 0 & 0 & 1.000 & 1.000 \\
& $(3,4),(4,1)$ & & & \\
13 & $(0,1),(0,2),(0,3),(0,4),(1,0),(2,0),(3,0),(4,0),(1,4),(2,3)$, & 0 & 0 & 1.157 & 1.001 \\
& $(3,1),(3,4),(4,2)$ & & & \\
14 & $(0,1),(0,2),(0,3),(0,4),(1,0),(2,0),(3,0),(4,0),(1,2),(1,3)$, & 0 & 0 & & \\
& $(2,3),(3,4),(4,1),(4,2)$ & & & \\
\end{tabular}

\begin{tabular}{|c|c|c|c|c|c|}
\hline$a$ & Design & nodd1 & nodd2 & rmtm0 & rmtm1 \\
\hline \multicolumn{6}{|c|}{$v=5$} \\
\hline 6 & $(0,1),(0,2),(3,0),(4,0),(2,5),(5,4)$ & 2 & $15^{\mathrm{a}}$ & 1.667 & 1.010 \\
\hline 7 & $(0,1),(0,2),(3,0),(4,0),(1,4),(2,5),(5,3)$ & 0 & 9 & 1.500 & 1.007 \\
\hline 8 & $(0,1),(0,2),(3,0),(4,0),(1,5),(2,5),(5,3),(5,4)$ & 0 & 4 & 1.250 & 1.003 \\
\hline 9 & $(0,1),(0,2),(0,3),(4,0),(5,0),(1,4),(1,5),(2,4),(3,5)$ & 0 & 2 & 1.366 & 1.003 \\
\hline 10 & $(0,1),(0,2),(3,0),(4,0),(5,0),(1,2),(1,4),(2,5),(3,4),(5,3)$ & 0 & 0 & 1.050 & 1.001 \\
\hline 11 & $\begin{array}{l}(0,1),(0,2),(3,0),(4,0),(5,0),(1,4),(1,5),(2,4),(2,5),(4,3), \\
(5,3)\end{array}$ & 0 & 0 & 1.229 & 1.002 \\
\hline 12 & $\begin{array}{l}(0,1),(0,2),(3,0),(4,0),(5,0),(1,3),(1,5),(2,3),(2,4),(3,5), \\
(4,1),(5,2)\end{array}$ & 0 & 0 & 1.223 & 1.002 \\
\hline 13 & $\begin{array}{l}(0,1),(0,2),(0,3),(0,4),(0,5),(1,0),(2,0),(3,0),(4,0),(5,0), \\
(1,4),(5,2),(3,5)\end{array}$ & 0 & 0 & 1.268 & 1.002 \\
\hline 14 & $\begin{array}{l}(0,1),(0,2),(0,3),(0,4),(0,5),(1,0),(2,0),(3,0),(4,0),(5,0), \\
(1,5),(2,4),(3,1),(5,2)\end{array}$ & 0 & 0 & 1.299 & 1.002 \\
\hline 15 & $\begin{array}{l}(0,1),(0,2),(0,3),(0,4),(0,5),(1,0),(2,0),(3,0),(4,0),(5,0), \\
(1,2),(2,3),(3,4),(4,5),(5,1)\end{array}$ & 0 & 0 & 1.000 & 1.000 \\
\hline 16 & $\begin{array}{l}(0,1),(0,2),(0,3),(0,4),(0,5),(1,0),(2,0),(3,0),(4,0),(5,0), \\
(1,5),(2,5),(3,4),(4,1),(4,2),(5,3)\end{array}$ & 0 & 0 & 1.164 & 1.001 \\
\hline 17 & $\begin{array}{l}(0,1),(0,2),(0,3),(0,4),(0,5),(1,0),(2,0),(3,0),(4,0),(5,0), \\
(1,3),(1,5),(2,3),(2,4),(3,5),(4,1),(5,2)\end{array}$ & 0 & 0 & 1.172 & 1.001 \\
\hline 18 & $\begin{array}{l}(0,1),(0,2),(0,3),(0,4),(0,5),(1,0),(2,0),(3,0),(4,0),(5,0), \\
(1,3),(1,4),(2,3),(2,4),(3,5),(4,5),(5,1),(5,2)\end{array}$ & 0 & 0 & 1.139 & 1.001 \\
\hline 19 & $\begin{array}{l}(0,1),(0,2),(0,3),(0,4),(0,5),(1,0),(2,0),(3,0),(4,0),(5,0), \\
(1,3),(2,3),(2,4),(3,4),(3,5),(4,1),(4,5),(5,1),(5,2)\end{array}$ & 0 & 0 & 1.143 & 1.001 \\
\hline 20 & $\begin{array}{l}(0,1),(0,2),(0,3),(0,4),(0,5),(1,0),(2,0),(3,0),(4,0),(5,0), \\
(1,2),(1,3),(2,3),(2,4),(3,4),(3,5),(4,1),(4,5),(5,1),(5,2)\end{array}$ & 0 & 0 & 1.000 & 1.000 \\
\hline
\end{tabular}




\begin{tabular}{|c|c|c|c|c|c|}
\hline$a$ & Design & nodd1 & nodd2 & $\mathrm{rmtm} 0$ & $\mathrm{rmtm} 1$ \\
\hline \multicolumn{6}{|c|}{$v=6$} \\
\hline 7 & $(0,1),(0,2),(3,0),(4,0),(5,0),(1,6),(6,3)$ & 3 & $21^{\mathrm{a}}$ & 1.667 & 1.010 \\
\hline 8 & $(0,1),(0,2),(3,0),(4,0),(5,0),(1,5),(2,6),(6,3)$ & 1 & 16 & 1.714 & 1.010 \\
\hline 9 & $(0,1),(0,2),(0,3),(4,0),(5,0),(6,0),(1,4),(2,5),(3,6)$ & 0 & 9 & 1.000 & 1.000 \\
\hline 10 & $(0,1),(0,2),(0,3),(4,0),(5,0),(1,6),(2,6),(3,6),(6,4),(6,5)$ & 0 & 5 & 1.450 & 1.004 \\
\hline 11 & $\begin{array}{l}(0,1),(0,2),(0,3),(4,0),(5,0),(6,0),(1,4),(1,5),(2,5),(2,6), \\
(3,6)\end{array}$ & 0 & 2 & 1.367 & 1.003 \\
\hline 12 & $\begin{array}{l}(0,1),(0,2),(0,3),(4,0),(5,0),(6,0),(1,4),(1,5),(2,4),(2,6), \\
(3,5),(3,6)\end{array}$ & 0 & 0 & 1.000 & 1.000 \\
\hline 13 & $\begin{array}{l}(0,1),(0,2),(0,3),(4,0),(5,0),(6,0),(1,4),(2,5),(2,6),(3,5), \\
(4,6),(5,1),(6,3)\end{array}$ & 0 & 0 & 1.227 & 1.002 \\
\hline 14 & $\begin{array}{l}(0,1),(0,2),(0,3),(4,0),(5,0),(6,0),(1,4),(1,6),(2,4),(2,5), \\
(3,5),(3,6),(5,1),(6,2)\end{array}$ & 0 & 0 & 1.246 & 1.002 \\
\hline 15 & $\begin{array}{l}(0,1),(0,2),(0,3),(4,0),(5,0),(6,0),(1,5),(1,6),(2,4),(2,6), \\
(3,4),(3,5),(4,1),(5,2),(6,3)\end{array}$ & 0 & 0 & 1.000 & 1.000 \\
\hline 16 & $\begin{array}{l}(0,1),(0,2),(0,3),(4,0),(5,0),(6,0),(1,5),(1,6),(2,4),(2,6), \\
(3,4),(3,5),(4,1),(4,6),(5,2),(6,3)\end{array}$ & 0 & 0 & 1.126 & 1.001 \\
\hline 17 & $\begin{array}{l}(0,1),(0,2),(0,3),(0,4),(0,5),(0,6),(1,0),(2,0),(3,0),(4,0), \\
(5,0),(6,0),(1,4),(2,6),(3,5),(5,2),(6,1)\end{array}$ & 0 & 0 & 1.294 & 1.002 \\
\hline 18 & $\begin{array}{l}(0,1),(0,2),(0,3),(0,4),(0,5),(0,6),(1,0),(2,0),(3,0),(4,0), \\
(5,0),(6,0),(1,2),(2,3),(3,4),(4,5),(5,6),(6,1)\end{array}$ & 0 & 0 & 1.000 & 1.000 \\
\hline 19 & $\begin{array}{l}(0,1),(0,2),(0,3),(0,4),(0,5),(0,6),(1,0),(2,0),(3,0),(4,0), \\
(5,0),(6,0),(1,4),(2,6),(3,5),(4,6),(5,1),(5,2),(6,3)\end{array}$ & 0 & 0 & 1.178 & 1.001 \\
\hline 20 & $\begin{array}{l}(0,1),(0,2),(0,3),(0,4),(0,5),(0,6),(1,0),(2,0),(3,0),(4,0), \\
(5,0),(6,0),(1,5),(1,6),(2,4),(2,6),(3,5),(4,1),(5,2),(6,3)\end{array}$ & 0 & 0 & 1.189 & 1.001 \\
\hline 21 & $\begin{array}{l}(0,1),(0,2),(0,3),(0,4),(0,5),(0,6),(1,0),(2,0),(3,0),(4,0), \\
(5,0),(6,0),(1,5),(1,6),(2,4),(2,6),(3,4),(3,5),(4,1),(5,2), \\
(6,3)\end{array}$ & 0 & 0 & 1.000 & 1.000 \\
\hline 22 & $\begin{array}{l}(0,1),(0,2),(0,3),(0,4),(0,5),(0,6),(1,0),(2,0),(3,0),(4,0), \\
(5,0),(6,0),(1,6),(2,4),(2,5),(3,4),(3,5),(4,1),(4,6),(5,1), \\
(6,2),(6,3)\end{array}$ & 0 & 0 & 1.148 & 1.001 \\
\hline 23 & $\begin{array}{l}(0,1),(0,2),(0,3),(0,4),(0,5),(0,6),(1,0),(2,0),(3,0),(4,0), \\
(5,0),(6,0),(1,3),(1,4),(2,4),(2,5),(3,2),(3,5),(4,6),(5,1), \\
(5,6),(6,1),(6,3)\end{array}$ & 0 & 0 & 1.143 & 1.001 \\
\hline 24 & $\begin{array}{l}(0,1),(0,2),(0,3),(0,4),(0,5),(0,6),(1,0),(2,0),(3,0),(4,0), \\
(5,0),(6,0),(1,3),(1,4),(2,3),(2,4),(3,5),(3,6),(4,5),(4,6), \\
(5,1),(5,2),(6,1)(6,2)\end{array}$ & 0 & 0 & 1.000 & 1.000 \\
\hline 25 & $\begin{array}{l}(0,1),(0,2),(0,3),(0,4),(0,5),(0,6),(1,0),(2,0),(3,0),(4,0), \\
(5,0),(6,0),(1,3),(1,4),(2,3),(2,4),(3,5),(3,6),(4,5),(4,6), \\
(5,1),(5,2),(5,6),(6,1),(6,2)\end{array}$ & 0 & 0 & 1.107 & 1.000 \\
\hline 26 & $\begin{array}{l}(0,1),(0,2),(0,3),(0,4),(0,5),(0,6),(1,0),(2,0),(3,0),(4,0), \\
(5,0),(6,0),(1,3),(1,4),(2,4),(2,5),(3,2),(3,4),(3,5),(4,5), \\
(4,6),(5,1),(5,6),(6,1),(6,2),(6,3)\end{array}$ & 0 & 0 & 1.108 & 1.000 \\
\hline 27 & $\begin{array}{l}(0,1),(0,2),(0,3),(0,4),(0,5),(0,6),(1,0),(2,0),(3,0),(4,0), \\
(5,0),(6,0),(1,2),(1,3),(1,4),(2,3),(2,4),(3,4),(3,5),(3,6), \\
(4,5),(4,6),(5,1),(5,2),(5,6),(6,1),(6,2)\end{array}$ & 0 & 0 & 1.000 & 1.000 \\
\hline
\end{tabular}




\begin{tabular}{|c|c|c|c|c|c|}
\hline$a$ & Design & nodd1 & nodd 2 & $\mathrm{rmtm} 0$ & rmtm1 \\
\hline \multicolumn{6}{|c|}{$v=7$} \\
\hline 8 & $(0,1),(0,2),(0,3),(4,0),(5,0),(6,0),(1,7),(7,6)$ & 4 & $28^{\mathrm{a}}$ & 1.667 & 1.010 \\
\hline 9 & $(0,1),(0,2),(0,3),(4,0),(5,0),(6,0),(1,6),(3,7),(7,5)$ & 2 & 24 & 1.714 & 1.010 \\
\hline 10 & $(0,1),(0,2),(0,3),(4,0),(5,0),(6,0),(7,0),(1,5),(2,6),(3,7)$ & 1 & 18 & 1.667 & 1.009 \\
\hline 11 & $\begin{array}{l}(0,1),(0,2),(0,3),(4,0),(5,0),(6,0),(1,6),(2,7),(3,7),(7,4), \\
(7,5)\end{array}$ & 0 & 7 & 1.333 & 1.003 \\
\hline 12 & $\begin{array}{l}(0,1),(0,2),(0,3),(4,0),(5,0),(6,0),(1,7),(2,7),(3,7),(7,4), \\
(7,5),(7,6)\end{array}$ & 0 & 6 & 1.750 & 1.005 \\
\hline 13 & $\begin{array}{l}(0,1),(0,2),(0,3),(4,0),(5,0),(6,0),(1,2),(1,6),(2,7),(3,7), \\
(5,6),(7,4),(7,5)\end{array}$ & 0 & 2 & 1.350 & 1.003 \\
\hline 14 & $\begin{array}{l}(0,1),(0,2),(0,3),(4,0),(5,0),(6,0),(7,0),(1,4),(1,5),(2,5), \\
(2,6),(3,6),(3,7),(4,7)\end{array}$ & 0 & 0 & 1.035 & 1.000 \\
\hline 15 & $\begin{array}{l}(0,1),(0,2),(0,3),(4,0),(5,0),(6,0),(7,0),(1,3),(1,6),(2,5), \\
(3,7),(4,6),(5,7),(6,2),(7,4)\end{array}$ & 0 & 0 & 1.241 & 1.002 \\
\hline 16 & $\begin{array}{l}(0,1),(0,2),(0,3),(4,0),(5,0),(6,0),(7,0),(1,7),(2,5),(2,6), \\
(3,6)(3,7),(4,1),(5,4),(6,4),(7,2)\end{array}$ & 0 & 0 & 1.254 & 1.002 \\
\hline 17 & $\begin{array}{l}(0,1),(0,2),(0,3),(4,0),(5,0),(6,0),(7,0),(1,4),(1,7),(2,5), \\
(2,7),(3,5),(3,6),(4,6),(5,1),(6,2),(7,3)\end{array}$ & 0 & 0 & 1.259 & 1.002 \\
\hline 18 & $\begin{array}{l}(0,1),(0,2),(0,3),(4,0),(5,0),(6,0),(7,0),(1,6),(1,7),(2,5), \\
(2,7),(3,5),(3,6),(4,1),(5,4),(6,2),(7,3),(7,4)\end{array}$ & 0 & 0 & 1.145 & 1.001 \\
\hline 19 & $\begin{array}{l}(0,1),(0,2),(0,3),(4,0),(5,0),(6,0),(7,0),(1,5),(1,6),(2,5), \\
(2,7),(3,5),(3,6),(4,7),(5,4),(6,2),(6,4),(7,1),(7,3)\end{array}$ & 0 & 0 & 1.147 & 1.001 \\
\hline 20 & $\begin{array}{l}(0,1),(0,2),(0,3),(0,4),(0,5),(0,6),(0,7),(1,0),(2,0),(3,0), \\
(4,0),(5,0),(6,0),(7,0),(1,6),(2,5),(3,7),(4,1),(6,3),(7,2)\end{array}$ & 0 & 0 & 1.292 & 1.002 \\
\hline 21 & $\begin{array}{l}(0,1),(0,2),(0,3),(0,4),(0,5),(0,6),(0,7),(1,0),(2,0),(3,0), \\
(4,0),(5,0),(6,0),(7,0),(1,2),(2,3),(3,4),(4,5),(5,6),(6,7), \\
(7,1)\end{array}$ & 0 & 0 & 1.000 & 1.000 \\
\hline 22 & $\begin{array}{l}(0,1),(0,2),(0,3),(0,4),(0,5),(0,6),(0,7),(1,0),(2,0),(3,0), \\
(4,0),(5,0),(6,0),(7,0),(1,4),(2,5),(3,6),(4,7),(5,7),(6,1), \\
(6,2),(7,3)\end{array}$ & 0 & 0 & 1.184 & 1.001 \\
\hline 23 & $\begin{array}{l}(0,1),(0,2),(0,3),(0,4),(0,5),(0,6),(0,7),(1,0),(2,0),(3,0), \\
(4,0),(5,0),(6,0),(7,0),(1,4),(1,7),(2,5),(2,7),(3,6),(4,6), \\
(5,1),(6,2),(7,3)\end{array}$ & 0 & 0 & 1.193 & 1.001 \\
\hline 24 & $\begin{array}{l}(0,1),(0,2),(0,3),(0,4),(0,5),(0,6),(0,7),(1,0),(2,0),(3,0), \\
(4,0),(5,0),(6,0),(7,0),(1,4),(1,7),(2,5),(2,7),(3,5),(3,6), \\
(4,6),(5,1),(6,2),(7,3)\end{array}$ & 0 & 0 & 1.196 & 1.001 \\
\hline 25 & $\begin{array}{l}(0,1),(0,2),(0,3),(0,4),(0,5),(0,6),(0,7),(1,0),(2,0),(3,0), \\
(4,0),(5,0),(6,0),(7,0),(1,4),(1,7),(2,5),(2,7),(3,5),(3,6), \\
(4,6),(5,1),(6,2),(7,3),(7,4)\end{array}$ & 0 & 0 & 1.163 & 1.001 \\
\hline 26 & $\begin{array}{l}(0,1),(0,2),(0,3),(0,4),(0,5),(0,6),(0,7),(1,0),(2,0),(3,0), \\
(4,0),(5,0),(6,0),(7,0),(1,7),(2,6),(2,7),(3,5),(3,6),(4,5), \\
(5,1),(5,2),(6,1),(6,4),(7,3),(7,4)\end{array}$ & 0 & 0 & 1.153 & 1.001 \\
\hline 27 & $\begin{array}{l}(0,1),(0,2),(0,3),(0,4),(0,5),(0,6),(0,7),(1,0),(2,0),(3,0), \\
(4,0),(5,0),(6,0),(7,0),(1,6),(1,7),(2,4),(2,6),(3,4),(3,5), \\
(4,1),(4,7),(5,1),(5,2),(6,3),(7,2),(7,3)\end{array}$ & 0 & 0 & 1.153 & 1.001 \\
\hline 28 & $\begin{array}{l}(0,1),(0,2),(0,3),(0,4),(0,5),(0,6),(0,7),(1,0),(2,0),(3,0), \\
(4,0),(5,0),(6,0),(7,0),(1,3),(1,7),(2,4),(2,7),(3,5),(3,6), \\
(4,5),(4,6),(5,1),(5,2),(6,1),(6,2),(7,3),(7,4)\end{array}$ & 0 & 0 & 1.005 & 1.000 \\
\hline
\end{tabular}


29

\section{0}

31

$(0,1),(0,2),(0,3),(0,4),(0,5),(0,6),(0,7),(1,0),(2,0),(3,0)$,
$(4,0),(5,0),(6,0),(7,0),(1,3),(1,4),(1,7),(2,3),(2,4),(2,6)$,
$(3,5),(3,6),(4,5),(4,6),(5,1),(5,2),(5,7),(6,1),(6,7),(7,2)$,

$(0,1),(0,2),(0,3),(0,4),(0,5),(0,6),(0,7),(1,0),(2,0),(3,0)$, $(4,0),(5,0),(6,0),(7,0),(1,6),(1,7),(2,4),(2,5),(3,4),(3,5)$, $(4,1),(4,6),(4,7),(5,1),(5,7),(6,2),(6,3),(7,2),(7,3)$

$(0,1),(0,2),(0,3),(0,4),(0,5),(0,6),(0,7),(1,0),(2,0),(3,0)$, $(4,0),(5,0),(6,0),(7,0),(1,6),(1,7),(2,4),(2,5),(3,4),(3,5)$, $(4,1),(4,6),(4,7),(5,1),(5,6),(5,7),(6,2),(6,3),(7,2),(7,3)$ $(0,1),(0,2),(0,3),(0,4),(0,5),(0,6),(0,7),(1,0),(2,0),(3,0)$, $(4,0),(5,0),(6,0),(7,0),(1,3),(1,4),(1,7),(2,3),(2,4),(2,7)$, $(3,5),(3,6),(4,5),(4,6),(5,1),(5,2),(5,7),(6,1),(6,2),(7,3)$, $(7,4)$ $(7,3),(7,4)$

33

$(0,1),(0,2),(0,3),(0,4),(0,5),(0,6),(0,7),(1,0),(2,0),(3,0)$, $(4,0),(5,0),(6,0),(7,0),(1,3),(1,4),(1,7),(2,3),(2,4),(2,5)$, $(3,5),(3,6),(4,5),(4,6),(5,1),(5,6),(5,7),(6,1),(6,2),(6,7)$, $(7,2),(7,3),(7,4)$ $(4,0),(5,0),(6,0),(7,0),(1,3),(1,4),(2,3),(2,4),(2,5),(3,4)$, $(3,5),(3,6),(4,5),(4,6),(4,7),(5,1),(5,6),(5,7),(6,1),(6,2)$, $(6,7),(7,1),(7,2),(7,3)$

\footnotetext{
a All the $\left(\begin{array}{l}a \\ 2\end{array}\right)$ "flawed" designs are disconnected because the number of slides $(a-2)$ in these designs is less than the number of the control versus test comparisons $v$.
}

\section{References}

Bechhofer, B.E., Tamhane, A.C., 1981. Incomplete block designs for comparing treatments with a control: general theory. Technometrics 23 , 45-57.

Graybill, F.A., 1983. Matrices with Applications in Statistics. second ed. Wadsworth, CA, USA.

Glonek, G.F., Solomon, P.J., 2004. Factorial and time course designs for cDNA microarray experiments. Biostatistics 5, 89-111.

Hedayat, A.S., Jacroux, M., Majumdar, D., 1988. Optimal designs for comparing test treatments with controls. Statist. Sci. 3, $462-491$.

Jacroux, M., 1989. The A-optimality of block designs for comparing test treatments with a control. J. Amer. Statist. Assoc. 84, $310-317$.

Kerr, M.K., Churchill, G.A., 2001. Experimental design for gene expression microarrays. Biostatistics 2, 183-201.

Landgrebe, J., Bretz, F., Brunner, E., 2006. Efficient design and analysis of two colour factorial microarray experiments. Comput. Statist. Data Anal. $50,499-517$.

McKay, B., 1991. "Nauty", "makeg” and "geng” C-programs available at 〈http://cs.anu.edu.au/people/ bdm/nauty/〉.

Speed, T.P., Yang, Y.H., 2002. Direct versus indirect designs for cDNA microarray experiments. Sankhya Ser. A 64 (3), $707-721$.

Ture, T.E., 1994. Optimal row-column designs for multiple comparisons with a control: a complete catalog. Technometrics 36, $292-299$.

Yang, Y.H., Speed, T.P., 2002. Design issues for cDNA microarray experiments. Nat. Rev. Genet. 3, 579-587. 